\title{
ON THE ZEROS OF CERTAIN RATIONAL FUNCTIONS*
}

\author{
BY
}

\author{
MORRIS MARDEN $\dagger$
}

I

1. Our present note uses simple methods to obtain zero-free regions for certain functions of the type

$$
F(z)=\sum \alpha_{j} f_{j}(z)
$$

where $\alpha_{j}$ is a complex number and $f_{j}(z)$ is a rational function of which the approximate positions of the zeros and poles are known. As first theorem we give what is a generalization not only of some of our recent results $\ddagger$ for the case that $f_{j}(z)=\left(z-a_{j}\right)^{-1}$, but also of some of Nagy's results $\S$ for the case that the $\alpha_{i}$ are real and positive.

THEOREM 1. Let $\alpha_{j}$ be complex numbers situated in the same given angular domain of which the vertex is the origin and the aperture is $\gamma, 0 \leqq \gamma<\pi$. Suppose that $m$ and $n$ are two integers independent of $j$, and that, for all values of $j$ and $k, a_{j k}$ and $b_{j k}$ are points of the same given convex region $K . \prod$

Then the locus of the zeros of the function

where

$$
F(z)=\sum_{1}^{p} \alpha_{j} f_{j}(z)
$$

$$
f_{j}(z) \equiv \frac{\left(z-a_{j 1}\right)\left(z-a_{j 2}\right) \cdots\left(z-a_{j m}\right)}{\left(z-b_{j 1}\right)\left(z-b_{j 2}\right) \cdots\left(z-b_{j n}\right)}
$$

is a region $S$ consisting of all the points at which $K$ subtends an angle $\phi$,

$$
\phi \geqq\left(\pi^{\cdot}-\gamma\right) /(m+n) \text {. }
$$

Several notions entering in the statement of this theorem and its proof need to be defined. By the locus of the zeros of $F$ we mean a region outside of

\footnotetext{
* Presented to the Society, December 30, 1929; received by the editors March 22, 1930. The author wishes to thank Professor Polya for the latter's many helpful suggestions and criticisms.

$\dagger$ National Research Fellow.

$\ddagger$ M. Marden, Bulletin of the American Mathematical Society, vol. 35 (1929), pp. 363-370.

8 J.v. S. Nagy, Acta Universitatis Hungaricae Francisco-Josephinae, vol. 1 (1923), pp. 127-141.

I The theorem and discussion that follow obtain also for convex regions $K$ that are infinite. Any of the points $M_{1}, M_{2}, N_{1}, N_{2}$ entering in the definition of "subtended angle" as given below may then be at infinity.
} 
which no point is a zero of $F$ and inside of which any point can be made a zero of $F$ through a suitable choice of the numbers $\alpha_{j}$ and of the points $a_{j k}$ and $b_{j k}$. By the symbol $\Varangle P_{1} P P_{2}$ we shall mean throughout this paper the angle measured in the counterclockwise sense from the ray $P P_{1}$ to the ray $P P_{2}$. Finally, by the angle subtended by the region $K$ at a point $P$ outside of or on the boundary of $K$, we mean the angle $\phi, \phi \leqq \pi$, such that for every pair of points $M_{1}, M_{2}$ in $K$ (with the subscripts so chosen that $\Varangle M_{1} P M_{2}$ does not exceed $\pi$ )

$$
\Varangle M_{1} P M_{2} \leqq \phi,
$$

and such that for at least two points $N_{1}, N_{2}$ in $K$

$$
\Varangle N_{1} P N_{2}=\phi .
$$

If $P$ is an interior point of $K$, the angle subtended by $K$ at $P$ will be taken as $\pi$.

On the basis of the preceding definitions let us investigate some of the properties of the region $S$, the ensemble of all the points at which $K$ subtends an angle $\phi, \phi \geqq \delta$, where $\delta \equiv(\pi-\gamma) /(m+n)$. Clearly $S$ contains $K$. If $P$ is an arbitrary point of $S$, and $Q$ an arbitrary point of $K$, every point of the line-segment $P Q$ is also included in the region $S$. This fact is evident if $P$ lies in $K$, for the region $K$ was assumed to be convex. If $P$ is not in $K$ and $R$ is an arbitrary point of the segment $P Q$, we shall denote by $M_{1}^{\prime}$ and $M_{2}^{\prime}$ the points of intersection with the line $N_{1} N_{2}$ of the lines through $R$ parallel to the lines $P N_{1}$ and $P N_{2}$. As $K$ is a convex region, the segment $N_{1} N_{2}$ and therefore the points $M_{1}^{\prime}$ and $M_{2}^{\prime}$ lie in $K$. This means, if $\phi$ and $\psi$ are the angles subtended by $K$ at $P$ and $R$ respectively, that

$$
\psi \geqq \phi \geqq \delta,
$$

and that hence $R$ is a point of $S$. In other words, the region $S$ is star-shaped with respect to every point of $K$. $^{*}$

Inequality (1) leads us to a further descriptive property of $S$. The region $S$, which for $\gamma=m=n-1=0$ is $K$ itself, expands as $\gamma$ and $m+n$ take on larger values and becomes the entire plane when either $\gamma$ approaches $\pi$ or $m+n$ becomes infinite.

2. To prove Theorem 1, we must establish the two propositions (a) that no point outside of $S$ is a zero of $F$ and (b) that any point in $S$ is a zero of $F$ for appropriately chosen numbers $\alpha_{j}$ and functions $f_{j}(z)$.

(a) Let $P: z$ be an arbitrary point outside of $K$; let $\phi$ be the angle subtended at $P$ by $K$; let $N_{1}$ and $N_{2}$ be two points of $K$ such that $\Varangle N_{1} P N_{2}=\phi$, and let $\pi+\tau$ be the angle made by the ray $P N_{1}$ with the positive real axis.

* A proof of this property may also be found on p. 128 of Nagy's article. 
Since the points $a_{j k}$ and $b_{j k}$ lie in the region $K$, the quantities $\left(z-a_{j k}\right)$ and $\left(z-b_{j k}\right)^{-1}$ are vectors drawn to $P$ having the properties

$$
\begin{aligned}
\tau & \leqq \operatorname{arc}\left(z-a_{j k}\right) \leqq \tau+\phi, \\
-(\tau+\phi) & \leqq \operatorname{arc}\left(z-b_{j k}\right)^{-1} \leqq-\tau .
\end{aligned}
$$

Hence,

$$
(m-n) \tau-n \phi \leqq \operatorname{arc} f_{j}(z) \leqq(m-n) \tau+m \phi .
$$

If, consequently, we suppose that for all $j$

we find that

$$
\kappa \leqq \operatorname{arc} \alpha_{j} \leqq \kappa+\gamma,
$$

$$
(m-n) \tau-n \phi+\kappa \leqq \operatorname{arc} \alpha_{j} f_{j}(z) \leqq(m-n) \tau+m \phi+\kappa+\gamma .
$$

For the point $P$ to be a zero of $F$ it is necessary that the difference between the extreme members of the latter inequality be at least $\pi$. That is to say, the point $P$ cannot be a zero of $F$ unless $\phi \geqq \delta, \delta \equiv(\pi-\gamma) /(m+n)$, i.e., unless $P$ lies in $S$.

(b) For the second part of the proof, let us assume $P: \zeta$ to be an arbitrary point of $S$ and $\phi$ to be the angle subtended at $P$ by $K$. Because $\phi \geqq \delta$, there exist in $K$ two points $Q_{1}: c_{1}, Q_{2}: c_{2}$ for which $\Varangle Q_{1} P Q_{2}=\delta$. Let $d_{1}$ and $d_{2}$ denote the distances from $P$ of the points $Q_{1}$ and $Q_{2}$ respectively, and let $\sigma$ denote the angle formed by the ray $P Q_{1}$ with the positive real axis. Let furthermore

$$
t_{1} \equiv\left[\left(\zeta-c_{1}\right) d_{1}^{-1}\right]^{m+n} \quad \text { and } \quad t_{2} \equiv\left[\left(\zeta-c_{2}\right) d_{2}^{-1}\right]^{m+n} e^{\gamma i}
$$

Then, as

$$
\begin{aligned}
& \operatorname{arc} t_{1}=(m+n) \sigma, \\
& \operatorname{arc} t_{2}=(m+n)(\sigma+\delta)+\gamma=\pi+(m+n) \sigma,
\end{aligned}
$$

the vectors $t_{1}$ and $t_{2}$ are equal and opposite and thus

$$
t_{1}+t_{2}=0 \text {. }
$$

Now consider the function

$$
G(z)=\frac{d_{2}^{n}\left(z-c_{1}\right)^{m}}{d_{1}{ }^{m}\left(z-c_{2}\right)^{n}}+\frac{e^{\gamma i} d_{1}^{n}\left(z-c_{2}\right)^{m}}{d_{2}^{m}\left(z-c_{1}\right)^{n}} .
$$

It has the point $P$ as a zero and it satisfies all the hypotheses of the theorem. Proposition (b) is therefore affirmed and with it the proof of Theorem 1 completed.

3. We shall now present a number of special cases and corollaries to the theorem.

(a) On our setting $\gamma=0$, Theorem 1 reduces to Nagy's general theorem 
(Nagy, p. 131), and on our placing $\gamma=m=n-1=0$ it becomes Gauss's theorem for the derivative of a polynomial.

(b) If $f_{j}(z)=z-a_{j}$, the zero of $F$ may be thought of as the "center of gravity" of the system of complex "masses," the mass $\alpha_{j}$ being at the point $a_{i}$. Thus, if all the points $a_{j}$ lie in the convex region $K$, their center of gravity lies in $S$, the region consisting of all points at which $K$ subtends an angle $\phi$, $\phi \geqq \pi-\gamma$. In particular, if all the $\alpha_{j}$ are real, $\gamma=0$ and $S$ coincides with $K$.

(c) Let us specialize $K$ to be in turn the ray $\theta=0$ (the positive real axis), the line-segment $A B$, and a circle $C$ of radius $r$.

In the first instance, the region $S$ is the angular domain $|\theta| \leqq \pi-\delta$.

In the second instance, $S$ is a region bounded by two circular arcs. These arcs are symmetric in the line $A B$ and cut one another in the points $A$ and $B$ at an angle of $2 \delta$. The points $A$ and $B$ are however not to be included in $S$. If $\gamma-\pi / 2=m=n-1=0$, the region $S$ becomes the interior and circumference of the circle having segment $A B$ as diameter-a result found also on page 366 of our previous note.

In the third instance, $S$ is the interior and circumference of the circle concentric with $C$ and of radius $r / \sin (\delta / 2)$. For $\gamma-\pi / 2=m=n-1=0$, this result coincides with that on page 366 of our previous note.

For the special case that $K$ is a polygonal region we refer to the discussion on pages 128-9 of Nagy's article.

(d) By virtue of Theorem 1, we may modify Nagy's generalization of Jentzsch's theorem so that it will read as follows:

Let $\alpha_{1}$ and $\alpha_{2}$ be two complex numbers situated in the same given angular domain of which the vertex is the origin and the aperture is $\gamma, 0 \leqq \gamma<\pi$. Let $f(z)$ be a polynomial of degree $n$ and $g_{1}(z)$ and $g_{2}(z)$ polynomials each of degree at most $m, m \leqq n-1$. Then, if all the zeros of the two functions

$$
F_{1}(z)=f(z)-g_{1}(z) \text { and } F_{2}(z)=f(z)-g_{2}(z)
$$

lie in the same given convex region $K$, the zeros of the function

$$
F(z)=f(z)-\frac{\alpha_{1} g_{1}(z)+\alpha_{2} g_{2}(z)}{\alpha_{1}+\alpha_{2}}
$$

will lie in a region $S$ consisting of all points at which $K$ subtends an angle $\phi$, $\phi \geqq(\pi-\gamma) /(m+1)$.

We have given this theorem without proof, as a proof can be constructed in close analogy with that given by Nagy (his article, p. 138) for his generalization of Jentzsch's theorem. 
(e) Let us next suppose that

$$
f_{j}(z) \equiv\left(1-\frac{\lambda_{j}}{n} z\right)^{-n}
$$

where the $\lambda_{i}$ are real numbers with $0<\lambda_{i}<\lambda_{i+1}$. As we saw in part (a) of this section, there are no zeros in the angular domain which is bounded by the rays $L_{1}^{\prime}$ and $L_{2}^{\prime}$ drawn from the point $z=n / \lambda_{p}$ at an inclination of $\delta$ with the positive real axis, and which does not include the points $z=n / \lambda_{j}$. The lines $L_{1}^{\prime}$ and $L_{2}^{\prime}$ have $y$-intercepts of magnitude $l^{\prime}=n \lambda_{p}^{-1} \tan \delta$. Now, when $n$ becomes infinite, the function $f_{j}(z)$ approaches $e^{\lambda_{j} z}$; the quantity $l^{\prime}$ approacines $l=\lambda_{p}^{-1}(\pi-\gamma)$ and the lines $L_{1}^{\prime}$ and $L_{2}^{\prime}$ become the parallels $L_{1}$ and $L_{2}$ to the $x$-axis at a distance $l$ above and below the $x$-axis. In the strip between $L_{1}$ and $L_{2}$ there are consequently no zeros of the function

$$
\Phi=\sum_{1}^{p} \alpha_{i} e^{\lambda_{j} z} .
$$

This result may, of course, be deduced with ease directly from the function $\Phi$.

(f) Finally, we notice the result described in Theorem 1 to be independent of the number of terms entering in the sum $F$ and therefore to be valid for certain infinite sums. This fact permits us, for instance, to state the following theorem.

Given the regular curve $\Gamma$ and the convex region $K$. Let $\alpha(t)$ be a complex function such that for all points $t$ on $\Gamma$

$$
\kappa \leqq \operatorname{arc}[\alpha(t) d t] \leqq \kappa+\gamma<\kappa+\pi .
$$

Let $a_{j}(t)$ and $b_{j}(t)$ be complex functions such that for all $j$ and for all points $t$ on $\Gamma$ the points $u_{j}$ and $v_{j}, u_{j} \equiv a_{j}(t)$ and $v_{j} \equiv b_{j}(t)$, lie in $K$. Then the integral

where

$$
I(z)=\int_{\Gamma} \alpha(t) f(z, t) d t,
$$

$$
f(z, t) \equiv \frac{\prod_{j=1}^{m}\left(z-a_{j}(t)\right)}{\prod_{j=1}^{n}\left(z-b_{j}(t)\right)},
$$

if it exists, has no zeros in the region $S$ consisting of all the points at which $K$ subtends an angle $\phi, \phi<(\pi-\gamma) /(m+n)$.

This theorem may be derived directly from Theorem 1 by dividing $\Gamma$ into $p$ parts by means of the successive points $t_{i}$, by setting $\Delta t_{j} \equiv t_{j+1}-t_{j}, \alpha_{j} \equiv \alpha\left(t_{j}\right) \Delta t_{j}$, and $f_{j}(z) \equiv f\left(z, t_{j}\right)$, and by finally letting $p$ become infinite. 
The theorem has as immediate consequence one concerning the $n$th derivative of certain analytic functions. If $K$ is bounded by the curve $\Gamma$ and the function $\psi(z)$ is analytic outside of $K$ (including the point at infinity) and continuous on $\Gamma$, the $n$th derivative of $\psi(z)$ has at a point $z$ outside of $K$ the value

$$
\psi^{(n)}(z)=-\frac{n !}{2 \pi i} \int_{\Gamma} \frac{\psi(t) d t}{(t-z)^{n+1}} .
$$

As the latter integral is of the type considered above, the ensuing theorem is true.

Given a convex region $K$, bounded by a closed, regular curve $\Gamma$. Let $\psi(z)$ be a function which is analytic outside of $K$ (including the point at infinity) and continuous on $\Gamma$, and which at every point $t$ of $\Gamma$ has the property

$$
\kappa \leqq \operatorname{arc}[\psi(t) d t] \leqq \kappa+\gamma<\kappa+\pi .
$$

Then the nth derivative of $\psi(z)$ has no zeros in the region $T$ consisting of all the points at which $K$ subtends an angle $\phi, \phi<(\pi-\gamma) /(n+1)$.

The zero-free regions $S$ and $T$ of the two preceding theorems do not, however, give the largest such regions. This is clear from the fact that the best approximation in Theorem 1 is attained only for sums $F$ of just two terms. (Cf. $\S 3$ (a).) When, for instance, $n=0$ and $K$ is a circle, a larger zero-free region than $T$ may be obtained directly. In this case

$$
\psi(t) d t \equiv i t \psi(t) d \theta
$$

where $\theta \equiv \operatorname{arc} t$. As $\psi(t) \cdot t$ is thus limited to an angular domain of opening less then $\pi$, it cannot increase by even $2 \pi$ when the point $t$ makes a complete circuit around $\Gamma$. Thus the entire exterior of $K$ is a zero-free region of $\psi^{(n)}(z)$.

\section{II}

4. Suppose $F_{1}$ and $F_{2}$ are two functions of the type considered in Part I of our present note. What can be said of the zeros of the difference $\Phi=F_{1}-F_{2}$ ? This question will be answered only in the case that $m+n=1$. We make this restriction expressly for the purpose of obtaining results which will have the same order of simplicity as those of Part I.

The theorem given below on the zeros of $\Phi$ involves the notion of the "inner angle" at a point $P$ of two non-overlapping convex regions $K$ and $L$. To explain this term we shall suppose $P$ to be an arbitrary point of the plane; $\theta$ and $\phi$ to be the angles subtended at $P$ by the regions $K$ and $L$ respectively, and $M_{1}, M_{2}$ to be a pair of points in $K$ and $N_{1}, N_{2}$ a pair of points in $L$ for which 


$$
\Varangle M_{1} P M_{2}=\theta \text { and } \Varangle N_{1} P N_{2}=\phi .
$$

Suppose the angles $M_{1} P M_{2}$ and $N_{1} P N_{2}$ do not overlap one another. Then the smaller of the two angles $M_{2} P N_{1}$ and $N_{2} P M_{1}$ is what we mean by the "inner angle at $P$ of the regions $K$ and $L$." The notion of inner angle will not be defined in the case that the angles $M_{1} P M_{2}$ and $N_{1} P N_{2}$ overlap one another.

Our theorem concerning the zeros of $\Phi$ is then the following:

THEOREM 2. Let $\alpha_{j}$ and $\beta_{k}$ be comblex numbers situated in the same given angular domain of which the vertex is the origin and the aperture is $\gamma, 0 \leqq \gamma<\pi$. Let $K$ and $L$ be two non-overlapping convex regions, in the first of which lie the points $a_{j}, j=1,2, \cdots, p$, and in the second of which lie the points $b_{k}, k=1$, $2, \cdots, q$. Suppose that $T$ is the ensemble of all points at which the inner angle of $K$ and $L$ is $\psi, \psi>\gamma$.

Then the locus of the zeros of the function*

$$
F(z)=\sum_{j=1}^{p} \frac{\alpha_{j}}{z-a_{j}}-\sum_{k=1}^{q} \frac{\beta_{k}}{z-b_{k}}
$$

is the region complementary to the region $T$.

5. The proof of this theorem, like that of Theorem 1, falls into two distinct parts.

(a) If we set

$$
G \equiv \sum_{j=1}^{p} \frac{\alpha_{j}}{a_{j}-z} \quad \text { and } \quad H \equiv \sum_{k=1}^{q} \frac{\beta_{k}}{b_{k}-z},
$$

we have as necessary condition for the point $P: z$ to be a zero of $F$ that the angular domain in which the vector $G$ may range overlap the one in which the vector $H$ may range.

We observe that multiplication of $F$ by the constant factor $e^{k i}$ does not affect the zeros of $F$. Without loss of generality we may therefore assume that

and

$$
-\pi / 2<-\gamma / 2 \leqq \operatorname{arc} \alpha_{j} \leqq \gamma / 2<\pi / 2
$$

$$
-\pi / 2<-\gamma / 2 \leqq \operatorname{arc} \beta_{k} \leqq \gamma / 2<\pi / 2 \text {. }
$$

Let us draw the lines $P Q_{1}, P Q_{2}, P R_{1}, P R_{2} ; P M_{1}^{\prime}, P M_{2}^{\prime}, P N_{1}^{\prime}, P N_{2}^{\prime}$,

* The theorem is also true for the function

$$
f(z)=\sum_{j=1}^{p} \alpha_{j}\left(z-a_{j}\right)-\sum_{k=1}^{q} \beta_{k}\left(z-b_{k}\right)
$$

whose zero is the "center of gravity" of the system of complex "masses," the mass $\alpha_{j}$ being at the point $a_{j}$ and the mass $-\beta_{k}$ being at the point $b_{k}$. For $\alpha_{j}$ and $\beta_{j}$ real and positive, the function $F(z)$ is the logarithmic derivative of a rational function.

† We are using this adjective to mean the region composed of all the points of the plane which are not in $T$. 
$P Q_{1}^{\prime}, P Q_{2}^{\prime}, P R_{1}{ }^{\prime}, P R_{2}^{\prime}$, where the notation of $\S 4$ is used, where $\Varangle Q_{1} P M_{1}$ $=\Varangle M_{2} P Q_{2}=\Varangle R_{1} P N_{1}=\Varangle N_{2} P R_{2}=\gamma / 2$, and where the primed letters denote points obtained by reflecting the corresponding unprimed lettered points in the line through $P$ parallel to the real axis.

As the points $a_{j}$ lie in $K$ and the points $b_{k}$ in $L$, the quantities $\left(a_{j}-z\right)^{-1}$ and $\left(b_{k}-z\right)^{-1}$ are vectors drawn from $P$, the first in the angle $M_{2}^{\prime} P M_{1}^{\prime}$ and the second in the angle $N_{2}^{\prime} P N_{1}^{\prime}$. The vectors $\alpha_{j}\left(a_{j}-z\right)^{-1}$ and $\beta_{k}\left(b_{k}-z\right)^{-1}$ are respectively in the angles $Q_{2}^{\prime} P Q_{1}^{\prime}$ and $R_{2}^{\prime} P R_{1}^{\prime}$. The vector $G$ therefore ranges in the angle $Q_{2}^{\prime} P Q_{1}^{\prime}$; the vector $H$, in the angle $R_{2}^{\prime} P R_{1}^{\prime}$.

Now, the angles $Q_{2}^{\prime} P Q_{1}^{\prime}$ and $R_{2}^{\prime} P R_{1}^{\prime}$ will overlap one another when and only when the angles $Q_{1} P Q_{2}$ and $R_{1} P R_{2}$ overlap one another. If the angles $M_{1} P M_{2}$ and $N_{1} P N_{2}$ overlap one another, the same will be true of the angles $Q_{1} P Q_{2}$ and $R_{1} P R_{2}$. If the angles $M_{1} P M_{2}$ and $N_{1} P N_{2}$ do not overlap one another, the angles $Q_{1} P Q_{2}$ and $R_{1} P R_{2}$ will overlap one another in and only in the case that the inner angle at $P$ does not exceed $\gamma$. This means that the zeros of $F$ must always lie in the region complementary to the region $T$.

(b) We need yet to show that conversely any point $P: \zeta$ of the complement to $T$ can be made a zero of $F$ through a suitable choice of the numbers $\alpha_{j}$ and $\beta_{k}$ and of the points $a_{j}$ and $b_{k}$.

As $P$ is a point of the complement of $T$, the angles $Q_{1} P Q_{2}$ and $R_{1} P R_{2}$ necessarily overlap one another. We shall suppose that they so overlap that the line $P R_{1}$ lies in the angular domain $Q_{1} P Q_{2}$. Let $P S, P U$ and $P V$ be rays drawn in the angular domains $R_{1} P Q_{2}, M_{1} P M_{2}$ and $N_{1} P N_{2}$ respectively, these rays being so chosen that $\Varangle U P S=\Varangle S P V=\gamma / 2$. Now on the line $P U$ exists a point $a$ of the region $K$ and on the line $P V$ exists a point $b$ of the region $L$. Denote the distance from $P$ of the point $a$ by $c$ and that of the point $b$ by $d$. Then the function

$$
F=\frac{c e^{-\gamma i / 2}}{z-a}-\frac{d e^{\gamma i / 2}}{z-b}
$$

has $P$ as a zero, for at $P$ it is a sum of two equal and opposite vectors. This function being one which satisfies the hypotheses of the theorem, the proof of Theorem 2 is now complete.

6. In this concluding section we shall try to throw light on the nature of the region $T$ of Theorem 2 through a study of some special cases.

Suppose, first, that $K$ consists merely of the point $A$, and $L$ merely of the point $B$. The region $T$, as the locus of the points $P$ where the angle $A P B$ exceeds $\gamma$, is the interior of the region $\Delta$ bounded by the two circular arcs which, symmetric in the line $A B$, cut one another in $A$ and $B$ at an angle of $2 \gamma$. The same region obtains for the case that $K$ and $L$ are two segments or 
half-rays along the same line, and $A$ and $B$ are the points of $K$ and $L$ respectively which are nearest together.

Suppose,-secondly, that $K$ is a circle with center at the point $C_{1}:(-a, 0)$ and of radius $r_{1}$, and $L$ is a circle with center at the point $C_{2}:(a, 0)$ and of radius $r_{2}$. Suppose the line-segment $C_{1} C_{2}$ to intersect circle $K$ in a point $A$ and circle $L$ in a point $B$.

To secure an upper limit to the region $T$, we concentrate at $A$ all the points $a_{j}$ and at $B$ all the points $b_{k}$. The new locus of the zeros of $F$ is clearly contained in the old and accordingly the new region $T^{\prime}$ encloses the old $T$. The region $T^{\prime}$ is precisely the interior of the region $\Delta$ described in the second paragraph of this section $(\$ 6)$.

For an accurate determination of the region $T$ we need to concern ourselves, on account of symmetry, only with the upper half-plane.

Let us first suppose $\gamma$ to be small. Then in the upper half-plane exist just one point $Q$ on the circle $K$ and just one point $R$ on the circle $L$ at which the inner angle is $\gamma$. The locus of the points outside of $K$ and $L$ at which the inner angle of $K$ and $L$ is $\gamma$ is then an $\operatorname{arc} \sigma$ of a curve which lies in the region $\Delta$ and connects the points $Q$ and $R$. If $T_{1}$ denotes the interior of the closed curve formed by joining together the $\operatorname{arc} \sigma$ with the $\operatorname{arc} Q A$ of circle $K$, with the $\operatorname{arc} R B$ of circle $L$ and with the line-segment $A B$, the region $T_{1}$ will comprise all the points of the upper half-plane at which the inner angle of $K$ and $L$ is defined and is greater than $\gamma$. The region $T$ is therefore composed of $T_{1}$ and of the reflection of $T_{1}$ in the line $A B$.

To obtain the equation of the curve $S$ of which the $\operatorname{arc} \sigma$ forms a part, we shall assume $P:(x, y)$ to be a point outside of $K$ and $L$, and $M$ and $N$ to be the points of contact with $K$ and $L$ respectively of those tangents from $P$ for which angle $M P N=\gamma$. In terms of the parameter $\theta \equiv \Varangle A C_{1} M$, the equations of $S$ are

$$
\begin{aligned}
(a+x) \cos \theta+y \sin \theta & =r_{1}, \\
(a-x) \cos (\gamma+\theta)-y \sin (\gamma+\theta) & =r_{2} .
\end{aligned}
$$

The parameter eliminated, the curve $S$ is represented by the single equation

$$
\begin{aligned}
{\left[\left(x^{2}+y^{2}-a^{2}\right) \sin \gamma\right.} & -2 a y]^{2}-\left[r_{1}(a-x) \sin \gamma+y\left(r_{1} \cos \gamma+r_{2}\right)\right]^{2} \\
& -\left[x\left(r_{2}+r_{1} \cos \gamma\right)+r_{1} y \sin \gamma+a\left(r_{2}-r_{1} \cos \gamma\right)\right]^{2}=0 .
\end{aligned}
$$

Hence $S$ is a bi-circular quartic. As application of the usual method shows, it is a bi-circular quartic with its two singular foci coincident in the point $z=i a \csc \gamma^{*}$

* In fact this curve has been considered in a slightly different connection by Walsh, who shows that it is a cartesian oval. See Quarterly Journal of Mathematics, vol. 50 (1924), pp. 154-165. 
What occurs when $\gamma$ approaches zero? The equation of $S$ becomes

$$
\left[4 a^{2}-\left(r_{1}+r_{2}\right)^{2}\right] y^{2}=\left[x\left(r_{1}+r_{2}\right)+a\left(r_{2}-r_{1}\right)\right]^{2},
$$

which factors into

$$
\pm y\left(4 a^{2}-\left(r_{1}+r_{2}\right)^{2}\right)^{1 / 2}=x\left(r_{1}+r_{2}\right)+a\left(r_{2}-r_{1}\right) .
$$

The bi-circular quartic thus degenerates into the two inner common tangents of the circles $K$ and $L$, and the points $Q$ and $R$ become the points of contact (in the upper half-plane) of these tangents with $K$ and $L$ respectively. The region $T$ is now the interior of the infinite region bounded by the line-segment $A B$, the $\operatorname{arc} Q A$ of circle $K$, the $\operatorname{arc} R B$. of circle $L$ and the rays along these inner common tangents from the points $Q$ and $R$ indefinitely outwards.

This result agrees with the deduction which, by allowing $m_{1}$ and $m_{2}$ to vary arbitrarily, one can make from a theorem due to Walsh.* We shall word this theorem as follows:

Let the numbers $\alpha_{j}$ and $\beta_{k}$ be real and positive, and the sums

$$
m_{1}=\sum_{1}^{p} \alpha_{j} \text { and } m_{2}=\sum_{1}^{q} \beta_{k}
$$

be such that $m_{1}>m_{2}$. Suppose the points $a_{j}$ to lie in a circle $K$ with center $\xi_{1}$ and of radius $r_{1}$, and the points $b_{k}$ to lie in a circle $L$ with center $\xi_{2}$ and of radius $r_{2}$. Then all the finite zeros of the function

$$
\sum_{1}^{p} \frac{\alpha_{j}}{z-a_{j}}-\sum_{1}^{q} \frac{\beta_{k}}{z-b_{k}}
$$

lie in a circle whose center is $\left(m_{1}-m_{2}\right)^{-1}\left(m_{1} \xi_{2}-m_{2} \xi_{1}\right)$ and whose radius is $\left(m_{1}-m_{2}\right)^{-1}\left(m_{1} r_{2}+m_{2} r_{1}\right)$.

So much for the case that $\gamma$ is small. Let us now examine that in which $\gamma$ has nearly the value $\pi$. In this case there exists no point on the circle $K$, the circle $L$, or the segment $A B$ at which the inner angle equals $\gamma$. The curve $S$ is a closed curve lying in the upper half-plane within the region $\Delta$. Its equation has still the form given above. As to the region $T$, it consists of the interior of $S$ and of the interior of the reflection of $S$ in the line $A B$.

When $\gamma$ approaches $\pi$, the region $\Delta$ collapses into coincidence with the line-segment $A B$. As at no point of the segment $A B$ is the inner angle of $K$ and $L$ equal to $\gamma$ (we are assuming the product $r_{1} r_{2} \neq 0$ ), the closed curve $S$ must shrink to a point on the approach of $\gamma$ to a certain value $\gamma_{1}, \gamma_{1}<\pi$. For this value $\gamma=\gamma_{1}$, the region $T$, as the interior of $S$ and of the reflection of $S$ in $A B$, must disappear, and the locus of the zeros of $F$, as the region

\footnotetext{
* J. L. Walsh, these Transactions, vol. 22 (1921), bottom of p. 114.
} 
complementary to $T$, must become the entire plane. The entire plane is therefore the locus of the zeros of $F$ for values of $\gamma$ between $\gamma_{1}$ and $\pi$.

It is now intuitively clear what deformations the region $T$ undergoes as $\gamma$ varies from zero to $\pi$. The end points $Q$ and $R$ of the $\operatorname{arc} \sigma$ move down the circles $K$ and $L$ towards the points $A$ and $B$ respectively. Then $Q$ and $R$ draw closer together along the segment $A B$ until they reach coincidence. They coincide at the point of the segment $A B$ where the inner angle of $K$ and $L$ takes on its maximum $\gamma_{0}$. As $\gamma$ then changes from $\gamma_{0}$ to $\gamma_{1}$, the $\operatorname{arc} \sigma$ remains a closed curve in the upper half of the region $\Delta$. When finally $\gamma$ becomes equal to $\gamma_{1}$, the oval shrinks to a point, and then and thereafter $T$ contains no points.

Emgenössische Technische Hochschule, ZURICH, SWITZERLAND 